\title{
TWO TAUBERIAN THEOREMS FOR NONCONVOLUTION VOLTERRA INTEGRAL OPERATORS
}

\author{
GUSTAF GRIPENBERG
}

ABstract. Two sets of sufficient conditions on the kernel $k(t, s)$ are given so that one can prove that if $x$ is a bounded function such that

$$
\lim _{\substack{t \rightarrow \infty \\ \tau \rightarrow 0}}|x(t+\tau)-x(t)|=0 \text { and } \lim _{t \rightarrow \infty} \int_{0}^{t} k(t, s) x(s) d s
$$

exists, then $\lim _{t \rightarrow \infty} x(t)$ exists.

1. Introduction and statement of results. The purpose of this note is to give sufficient conditions on the kernel $k$ so that the following conclusion holds: If $x$ is a bounded function such that

$$
\lim _{\substack{t \rightarrow \infty \\ \tau \rightarrow 0}}|x(t+\tau)-x(t)|=0 \text { and } \lim _{t \rightarrow \infty} \int_{0}^{t} k(t, s) x(s) d s
$$

exists, then $\lim _{t \rightarrow \infty} x(t)$ exists.

In the case when the kernel is of convolution type, i.e. $k(t, s)=K(t-s)$ for some function $K$, and $K$ is integrable, then the answer is given by Pitt's form of Wiener's Tauberian theorem, see e.g. [4, p. 210]. The statement above holds if and only if $K^{\wedge}(y) \neq 0, y \in(-\infty, \infty)$ where "^" denotes the Fourier transform. The situation when $K$ is not integrable is studied in [2].

Since we do not make the assumption that the kernel $k$ is of convolution type, we cannot use the Fourier transform. Then it becomes difficult to find very general assumptions on $k$ that imply the desired conclusion, but as is seen from the two theorems below, more specialized conditions can be used.

Let $\Delta=\{(t, s) \mid 0 \leqslant s \leqslant t\}$.

THEOREM 1. Assume that the function $k$ is defined on $\Delta$ and that

$$
\begin{gathered}
\text { kis positive and continuous on } \Delta, \\
k(t, 0) \leqslant k(\tau, 0) \quad \text { when } 0 \leqslant \tau \leqslant t, \\
k\left(t_{1}, s_{1}\right) k\left(t_{2}, s_{2}\right) \leqslant k\left(t_{1}, s_{2}\right) k\left(t_{2}, s_{1}\right) \quad \text { when } u \leqslant s_{1} \leqslant s_{2} \leqslant t_{1} \leqslant t_{2},
\end{gathered}
$$

Received by the editors October 26, 1982.

1980 Mathematics Subject Classification. Primary 40E05, 45E99; Secondary 47G05. 


$$
\begin{gathered}
\lim _{t \rightarrow \infty} \int_{0}^{t} k(t, s) d s \leqslant+\infty \text { exists } \\
\lim _{t \rightarrow \infty} k(t, s)=0 \text { for all } s \text { if } \\
\lim _{t \rightarrow \infty} \int_{0}^{t} k(t, s) d s<\infty \text { and } \\
\lim _{t \rightarrow \infty}\left(\int_{0}^{t} k(t, s) d s\right)\left(1+\max _{\tau \in[0, t]} \int_{0}^{\tau} k(\tau, s) d s\right)^{-1}=1 \text { if } \\
\lim _{t \rightarrow \infty} \int_{0}^{t} k(t, s) d s=+\infty, \\
\liminf _{t \rightarrow \infty} \int_{t-h}^{t} k(t, s) d s>0 \text { for all } h>0 .
\end{gathered}
$$

If

$$
\begin{gathered}
x \in L^{\infty}\left(\mathbf{R}^{+} ; \mathbf{R}\right), \\
\lim _{\substack{t \rightarrow \infty \\
\tau \rightarrow 0}}|x(t+\tau)-x(t)|=0
\end{gathered}
$$

and

$$
\lim _{t \rightarrow \infty} \int_{0}^{t} k(t, s) x(s) d s=\alpha, \quad|\alpha|<\infty,
$$

then

$$
\lim _{t \rightarrow \infty} x(t)=\alpha\left(\lim _{t \rightarrow \infty} \int_{0}^{t} k(t, s) d s\right)^{-1} .
$$

Observe that if $k(t, s)=K(t-s)$, then the assumptions (1.1) and (1.2) imply that (1.4)-(1.6) hold and that

$$
\hat{K^{\wedge}}(y)=\lim _{x \rightarrow 0-} \int_{0}^{\infty} e^{-i(y+i x) t} K(t) d t \neq 0 .
$$

Note also that $k(t, s)$ is sufficiently smooth, then (1.3) is equivalent to $\partial / \partial s \partial / \partial t \log (k(t, s)) \leqslant 0$. To see that the assumption (1.6) cannot be dropped, consider the example $k(t, s)=(t+1)^{-1}$ and $x(t)=\cos (t)$. Then all the assumptions except (1.6) hold and (1.10) fails.

In the second theorem we do not make the assumption (1.3) that is crucial in Theorem 1.

THEOREM 2. Assume that $k$ is defined on $\Delta$ and that for each $t \geqslant 0$, the function: $s \rightarrow k(t, s), 0 \leqslant s \leqslant t$, is measurable and nonnegative,

$$
\begin{gathered}
k(t, s) \leqslant k(\tau, s) \quad \text { when } 0 \leqslant s \leqslant \tau \leqslant t, \\
\sup _{(t, s) \in \Delta} k(t, s)<\infty,
\end{gathered}
$$


there exists a measurable and nonnegative function $a$ on $R^{+}$such that $\int_{0}^{\infty} a(t) d t>0$ and

(1.14) $\liminf _{t \rightarrow \infty} \inf _{h \in(0,1]} \inf _{s \in[0, t]}\left(k(t, s)-k(t+h, s)-\int_{t-s}^{t+h-s} a(u) d u\right) \geqslant 0$,

$$
\lim _{t \rightarrow \infty} \int_{0}^{t} k(t, s) d s<\infty \text { exists }
$$

(1.16) $\lim _{\tau \rightarrow \infty} \limsup _{t \rightarrow \infty} \int_{0}^{t-\tau} k(t, s) d s<2^{-1}\left(\lim _{t \rightarrow \infty} \int_{0}^{t} k(t, s) d s+\int_{0}^{\infty} \lim _{t \rightarrow \infty} k(t, s) d s\right)$.

Then (1.7)-(1.9) imply

$$
\begin{aligned}
\lim _{t \rightarrow \infty} x(t)= & \left(\alpha-\int_{0}^{\infty} \lim _{t \rightarrow \infty} k(t, s) x(s) d s\right) \\
& \times\left(\lim _{t \rightarrow \infty} \int_{0}^{t} k(t, s) d s-\int_{0}^{\infty} \lim _{t \rightarrow \infty} k(t, s) d s\right)^{-1} .
\end{aligned}
$$

Note that by (1.11), (1.12) and (1.15) $\lim _{t \rightarrow \infty} k(t, s)$ exists and is a nonnegative integrable function. The assumption (1.14) is not quite the best possible one but if, for example, $k(t, s)=\chi_{[0,1]}(t-s), 0 \leqslant s \leqslant t$, then all the assumptions of Theorem 2 except (1.14) hold, and to see that (2.17) does not follow from (1.7)-(1.9) one can take $x(t)=\sin (2 \pi t)$.

In [2] it is shown that Theorem 2 fails in the convolution case if (1.15) and (1.16) are dropped.

2. Proof of Theorem 1. The proof will be based on the following lemma that has also been established in [1].

Lemмa 2.1. Let (1.1)-(1.5) hold. Then there exists a function $r$, defined on $\Delta$, such that

$$
\begin{aligned}
& r \text { is continuous on } \Delta \text { and } 0<r(t, s) \leqslant k(t, s), \quad(t, s) \in \Delta, \\
& k(t, s)-r(t, s)=\int_{s}^{t} k(t, u) r(u, s) d u=\int_{s}^{t} r(t, u) k(u, s) d u,
\end{aligned}
$$

$$
(t, s) \in \Delta
$$

$$
\int_{0}^{t} r(t, s) d s \leqslant 1, \quad t \geqslant 0
$$$$
\int_{\tau}^{t} r(t, s) d s \geqslant \int_{\tau}^{t} k(t, s) d s\left(1+\sup _{u \in[\tau, t]} \int_{\tau}^{u} k(u, s) d s\right)^{-1}, \quad 0 \leqslant \tau \leqslant t,
$$$$
\lim _{t \rightarrow \infty} \int_{0}^{T} r(t, s) d s=0 \text { for all } T>0 .
$$

Proof. By [3, Theorem 3.1, p. 202] and (1.1) there exists a continuous function $r$ on $\Delta$ such that the resolvent equation (2.2) holds. Let $0 \leqslant s_{0}<t_{0}$ be such that $r\left(t_{0}, s_{0}\right)=0$. From the continuity of $r$ and the fact that $r\left(s_{0}, s_{0}\right)=k\left(s_{0}, s_{0}\right)>0$ we see that we may assume that

$$
r\left(t, s_{0}\right)>0, \quad t \in\left(s_{0}, t_{0}\right) .
$$


By (1.1), (2.2) and the assumption that $r\left(t_{0}, s_{0}\right)=0$ we have

$$
\begin{aligned}
0= & k\left(t_{0}, s_{0}\right)-\left(k\left(t, s_{0}\right)\right)^{-1} k\left(t_{0}, s_{0}\right) \int_{s_{0}}^{t} k(t, u) r(u, s) d u \\
& +\int_{s_{0}}^{t}\left(\left(k\left(t, s_{0}\right)\right)^{-1} k\left(t_{0}, s_{0}\right) k(t, u)-k\left(t_{0}, u\right)\right) r\left(u, s_{0}\right) d u \\
& -\int_{t}^{t_{0}} k\left(t_{0}, u\right) r\left(u, s_{0}\right) d u
\end{aligned}
$$

for every $t \in\left(s_{0}, t_{0}\right)$. From this equality we conclude with the aid of (1.3), (2.2) and (2.6) that

$$
0 \geqslant\left(k\left(t, s_{0}\right)\right)^{-1} k\left(t_{0}, s_{0}\right) r\left(t, s_{0}\right)-\max _{u \in\left[t, t_{0}\right]} r\left(u, s_{0}\right) \int_{t}^{t_{0}} k\left(t_{0}, u\right) d u .
$$

Let $\left\{t_{n}\right\}_{n=1}^{\infty}$ be a sequence of numbers such that $t_{n} \rightarrow t_{0^{-}}$as $n \rightarrow \infty$ and such that $r\left(t_{n}, s_{0}\right)=\max _{u \in\left[t_{n}, t_{0}\right]} r\left(u, s_{0}\right)$ for all $n$. Then (2.7) gives a contradiction because $\left(k\left(\dot{t}_{n}, s_{0}\right)\right)^{-1} k\left(t_{0}, s_{0}\right) \rightarrow 1, \int_{t_{n}}^{t_{0}} k\left(t_{0}, u\right) d u \rightarrow 0$ as $n \rightarrow \infty$ and $r\left(t_{n}, s_{0}\right)>0$ for all $n$ by (2.6). Thus we have established the first inequality in (2.1) and the second one follows from the first one, (1.1) and (2.2).

Using (1.2), (2.1) and (2.2) we get

$$
\int_{0}^{t} r(t, u) d u \leqslant(k(t, 0))^{-1} \int_{0}^{t} r(t, u) k(u, 0) d u=1-r(t, 0)(k(t, 0))^{-1} \leqslant 1, \quad t \geqslant 0,
$$

so that (2.3) holds. From (2.2) and an application of Fubini's theorem we obtain

$$
\int_{\tau}^{t} r(t, s) d s=\int_{\tau}^{t} k(t, s) d s-\int_{\tau}^{t} r(t, u) \int_{\tau}^{u} k(u, s) d s d u
$$

and (2.4) follows immediately since $r$ is positive.

Since $k(t, t)$ is continuous, hence bounded on compact intervals, and $k(t, 0)$ is nonincreasing, see (1.2), it follows from (1.3) that

$$
\sup _{\substack{t \geq s \\ s \in[0, T]}} k(t, s)<\infty \quad \text { for all } T>0 .
$$

If $\lim _{t \rightarrow \infty} k(t, s)=0$ for all $s \geqslant 0$, then it follows from $(2.1),(2.8)$ and the dominated convergence theorem that $(2.5)$ holds. If

$$
\lim _{t \rightarrow \infty}\left(\int_{0}^{t} k(t, s) d s\right)\left(1+\max _{\tau \in[0, t]} \int_{0}^{\tau} k(\tau, s) d s\right)^{-1}=1
$$

then it follows from (2.8) that for all $T>0$,

$$
\lim _{t \rightarrow \infty}\left(\int_{T}^{t} k(t, s) d s\right)\left(1+\max _{\tau \in[T, t]} \int_{T}^{\tau} k(\tau, s) d s\right)^{-1}=1 .
$$

If we combine this fact with (2.3) and (2.4), then we see that (2.5) holds. This completes the proof of Lemma 2.1.

If we replace $x$ by $x(t)-\alpha\left(\lim _{t \rightarrow \infty} \int_{0}^{t} k(t, s) d s\right)^{-1}$, then we may assume that

$$
\alpha=0 \text { or } \lim _{t \rightarrow \infty} \int_{0}^{t} k(t, s) d s=+\infty \text {. }
$$


If (1.10) does not hold we can, therefore, by (1.8) assume that there exist numbers $\delta$, $\gamma>0$ and a sequence $\left\{t_{n}\right\}_{n=1}^{\infty}$ tending to $+\infty$ such that

$$
x(t) \geqslant \delta \quad \text { when } t \in\left[t_{n}, t_{n}+\gamma\right], \quad n=1,2, \ldots
$$

Since no assumptions are violated if we multiply $k(t, s)$ by a large positive number, by (1.6) we may assume that

$$
\int_{t_{n}}^{t_{n}+\gamma} k\left(t_{n}+\gamma, s\right) d s \geqslant 4\left(1+\|x\|_{L^{x}\left(R^{*}: R\right)}\right) \delta^{-1}, \quad n=1.2 \ldots \ldots
$$

From (2.2) we conclude that

$$
\int_{0}^{t} r(t, s) x(s) d s=\int_{0}^{t} k(t, s) x(s) d s-\int_{0}^{t} r(t, s) \int_{0}^{s} k(s, u) x(u) d u d s, \quad t \geqslant 0 .
$$

By (1.9), (2.1), (2.3), (2.5) and (2.12) we see that

$$
\lim _{t \rightarrow \infty} \int_{0}^{t} r(t, s) x(s) d s=0
$$

holds if $\alpha=0$ and, if $\alpha \neq 0$ we use the fact that in this case $\lim _{t \rightarrow \infty} \int_{0}^{t} r(t, s) d s=1$ by (1.5), (2.3), (2.4) and (2.9) and we derive (2.13) from (2.12).

Now we choose the numbers $\tau_{n}$ so that

$$
t_{n} \leqslant \tau_{n} \leqslant t_{n}+\gamma \text { and } \int_{t_{n}}^{\tau_{n}} k\left(\tau_{n}, s\right) d s=\max _{u \in\left[t_{n}, t_{n}+\gamma\right]} \int_{t_{n}}^{u} k(u, s) d s,
$$

for all $n$. Then it follows from (2.1), (2.3), (2.4), (2.10), (2.11) and (2.14) that

$$
\begin{aligned}
\int_{0}^{\tau_{n}} r\left(\tau_{n}, s\right) x(s) d s & \geqslant \tau \int_{t_{n}}^{\tau_{n}} r\left(\tau_{n}, s\right) d s-\|x\|_{L^{\infty}\left(R^{+}: R\right)}\left(1-\int_{t_{n}}^{\tau_{n}} r\left(\tau_{n}, s\right) d s\right) \\
& \geqslant\left(4 \delta+3 \delta\|x\|_{L^{\infty}\left(R^{+}: R\right)}\right)\left(\delta+4\left(1+\|x\|_{L^{\infty}\left(R^{+}: R\right)}\right)\right)^{-1}, \quad n=1,2, \ldots
\end{aligned}
$$

But this is a contradiction since (2.13) holds and the proof of Theorem 1 is completed.

3. Proof of Theorem 2. First we note that without loss of generality we may assume that

$$
\lim _{t \rightarrow \infty} k(t, s)=0 \quad \text { for all } s \geqslant 0 .
$$

It follows from (1.12) that $\lim _{t \rightarrow \infty} k(t, s) \stackrel{\text { def }}{=} b(s)$ exists and by (1.11) and (1.15) $b(s) \geqslant 0$ and $\int_{0}^{\infty} b(s) d s<\infty$. If we define $k_{0}(t, s)=k(t, s)-b(s)$, then we see that the function $k_{0}$ satisfies all the assumptions of Theorem 2 .

If $\alpha \neq 0$ in (1.9), then we subtract $\alpha\left(\lim _{t \rightarrow \infty} \int_{0}^{t} k(t, s) d s\right)^{-1}$ from $x$. Thus we assume that (1.7)-(1.9) hold with $\alpha=0$ but that (1.10) (or (1.17)) does not hold. No generality is lost if we suppose that

(3.2) $\delta_{+} \geqslant-\delta_{-}, \quad \delta_{+}>0 \quad$ where $\delta_{+}=\limsup _{t \rightarrow \infty} x(t)$ and $\delta_{-}=\liminf _{t \rightarrow \infty} x(t)$.

It follows from (1.14) that there exists a set $E$, with Lebesgue measure $m(E)>0$ such that $\int_{\tau}^{\tau+h} a(s) d s>0$ for all $h>0$ when $\tau \in E$. The following lemma is crucial for the proof. 
LeMma 3.1. If $\left\{t_{n}\right\}_{n=1}^{\infty}$ is a sequence of positive numbers tending to $+\infty$ such that $\lim _{n \rightarrow \infty} x\left(t_{n}\right)=\delta_{+}$, then $\lim _{n \rightarrow \infty} x\left(t_{n}-\tau\right)=\delta_{+}$for every $\tau \in E$.

Proof. Suppose that this is not the case for some $\tau \in E$ and some sequence $\left\{t_{n}\right\}_{n=1}^{\infty}$ tending to $+\infty$ such that $\lim _{n \rightarrow \infty} x\left(t_{n}\right)=\delta_{+}$. Then there exist by (1.8) and (3.2) (since we can pick a subsequence if necessary), numbers $\varepsilon, \gamma>0$ such that

(3.3) $x\left(t_{n}-\tau-s\right) \leqslant \delta_{+}-\varepsilon, \quad s \in[0, \gamma], \quad x\left(t_{n}\right)>\delta_{+}-\varepsilon, \quad n=1,2, \ldots$

Choose $T>0$ to be such that (see (1.13) and the definitions of $\delta_{+}$and $E$ ),

$$
\left(\sup _{(t, s) \in \Delta} k(t, s)\right)\left(\sup _{t \geqslant T} x(t)-\delta_{+}\right)<2^{-1} \varepsilon \int_{\tau}^{\tau+\gamma} a(s) d s .
$$

Next we choose a function $g$ with the following properties:

$$
g: R^{+} \rightarrow(0,1] \text { is nonincreasing, } \quad \lim _{t \rightarrow \infty} g(t)=0,
$$

$$
\liminf _{t \rightarrow \infty}(g(t))^{-1} \inf _{s \in[0, t-g(t)]}\left(k(t-g(t), s)-k(t, s)-\int_{t-g(t)-s}^{t-s} a(u) d u\right) \geqslant 0 .
$$

This is possible by (1.9), (1.13)-(1.15) and (3.1).

From (1.11), (1.12), (1.13) and (3.3) we obtain

$$
\begin{aligned}
& \int_{t_{n}-g\left(t_{n}\right)}^{t_{n}} k\left(t_{n}, s\right) x(s) d s \geqslant x\left(t_{n}\right) \int_{t_{n}-g\left(t_{n}\right)}^{t_{n}} k\left(t_{n}, s\right) d s \\
&-g\left(t_{n}\right)\left(\sup _{s \in\left[t_{n}-g\left(t_{n}\right), t_{n}\right]}\left|x(s)-x\left(t_{n}\right)\right|\right) \sup _{(t, s) \in \Delta} k(t, s), \\
& n=1,2, \ldots,
\end{aligned}
$$

and

$$
\begin{aligned}
\int_{0}^{t_{n}-g\left(t_{n}\right)} & \left(k\left(t_{n}, s\right)-k\left(t_{n}-g\left(t_{n}\right), s\right)\right) x(s) d s \\
\geqslant & x\left(t_{n}\right) \int_{0}^{t_{n}-g\left(t_{n}\right)}\left(k\left(t_{n}, s\right)-k\left(t_{n}-g\left(t_{n}\right), s\right)\right) d s \\
& +2\|x\|_{L^{\infty}\left(R^{+} ; R\right)} \int_{0}^{T}\left(k\left(t_{n}, s\right)-k\left(t_{n}-g\left(t_{n}\right), s\right)\right) d s+\sup _{t \geqslant T}\left(x(t)-x\left(t_{n}\right)\right) \\
& \times\left(\int_{0}^{t_{n}} k\left(t_{n}, s\right) d s-\int_{0}^{t_{n}-g\left(t_{n}\right)} k\left(t_{n}-g\left(t_{n}\right), s\right) d s-g\left(t_{n}\right) \sup _{(t, s) \in \Delta} k(t, s)\right) \\
& +\int_{t_{n}-\tau-\gamma}^{t_{n}-\tau}\left(k\left(t_{n}, s\right)-k\left(t_{n}-g\left(t_{n}\right), s\right)\right) d s\left(\delta_{+}-\varepsilon-x\left(t_{n}\right)\right),
\end{aligned}
$$


(We assume that $T_{n}$ is so large that $\tau>g\left(t_{n}\right)$.) Divide both sides of the inequalities in (3.8) and (3.9) by $g\left(t_{n}\right)$, add them up, let $n \rightarrow \infty$ and use (1.8) and (3.5)-(3.7). This yields

$$
0 \geqslant-\sup _{t \geqslant T}\left(x(t)-\delta_{+}\right) \sup _{(t, s) \in J} k(t, s)+\varepsilon \int_{\tau}^{\tau+\gamma} a(u) d u,
$$

so that we have a contradiction by (3.4). This completes the proof of Lemma 3.1.

By the definition of $\delta_{+}$there exists a sequence $\left\{t_{n}\right\}_{n=1}^{\infty}$ tending to $+\infty$ such that $\lim _{n \rightarrow \infty} x\left(t_{n}\right)=\delta_{+}$. If we now repeatedly use Lemma 3.1 and the fact that since $m(E)>0$ it follows that $\cup_{n=1}^{\infty}\left\{\sum_{i=1}^{n} s_{i} \mid s_{i} \in E, i=1, \ldots n\right\} \supset(T, \infty)$ for some $T>0$, then we conclude that there exists a sequence $\left\{t_{n}\right\}_{n=1}^{\infty}$ tending to $+\infty$ such that (3.10) $x\left(t+t_{n}\right) \rightarrow \delta_{+}$uniformly on compact subsets of $(-\infty, 0]$ as $n \rightarrow \infty$.

Since

$$
\limsup _{t \rightarrow \infty} \int_{0}^{t-\tau} k(t, s) d s=\limsup _{t \rightarrow \infty} \int_{0}^{t} k(t+\tau, s) d s
$$

it follows from (1.12) that the limit on the left-hand side in the inequality in (1.6) exists. From (1.15), (1.16), (3.1) and (3.2) we deduce that there exist positive numbers $\tau, T$ and $N$ such that

$$
\delta_{+} \inf _{n \geqslant N}\left(\int_{t_{n}-\tau}^{t_{n}} k\left(t_{n}, s\right) d s-\int_{0}^{t_{n}-\tau} k\left(t_{n}, s\right) d s\right)>\sup _{t \geqslant T}\left(\delta_{-}-x(t)\right) \sup _{t \geqslant 0} \int_{0}^{t} k(t, s) d s .
$$

Since $k(t, s) \geqslant 0$ we have

$$
\begin{aligned}
\int_{0}^{t_{n}} k\left(t_{n}, s\right) x(s) d s \geqslant & \delta_{+} \int_{t_{n}-\tau}^{t_{n}} k\left(t_{n}, s\right) d s-\sup _{s \in\left[t_{n}-\tau . t_{n}\right]}\left|x(t)-\delta_{+}\right| \sup _{t \geqslant 0} \int_{0}^{t} k(t, s) d s \\
& +\delta_{-} \int_{0}^{t_{n}-\tau} k\left(t_{n}, s\right) d s+\inf _{t \geqslant T}\left(x(t)-\delta_{-}\right) \sup _{t \geqslant 0} \int_{0}^{t} k(t, s) d s \\
& -2\|x\|_{L^{\infty}\left(R^{+}: R\right)} \int_{0}^{T} k\left(t_{n}, s\right) d s, \quad n=N, N+1, \ldots
\end{aligned}
$$

If we let $n \rightarrow \infty$ on both sides of this inequality, then we conclude from (1.9) (with $\alpha=0),(3.2),(3.10)$ and (3.11) that we have a contradiction because $\lim _{t \rightarrow \infty} \int_{0}^{T} k(t, s) d s=0$ by (1.13), (3.1) and the dominated convergence theorem. This completes the proof of Theorem 2 .

\section{REFERENCES}

1. G. Gripenberg, On Volterra equations with nonconvolution kernels, Report-HTKK-MAT-A118, Helsinki Univ. of Technology, 1978.

2. __ A Tauberian problem for a Volterra integral operator, Proc. Amer. Math. Soc. 84 (1981), $576-582$.

3. R. K. Miller, Nonlinear Volterra integral equations, Benjamin, New York, 1971.

4. D. V. Widder, The Laplace transform, Princeton Univ. Press, Princeton, N. J., 1946.

InStitute of Mathematics, Helsinki University OF TeChNOlOgy, SF-02150 EsPoo 15, FinLAND 